\title{
El investigador fragmentado: conflictos y tensiones derivados de la diversificación de su carga laboral
}

Elizabeth Ocampo-Gómez, Silvia Jiménez-García y Lilia Palacios-Ramírez

\section{RESUMEN}

La política de diversificación de la carga instaurada en los noventa dio auge a acciones encaminadas a la formación de redes y cuerpos académicos, la introducción del concepto de perfil de desempeño equilibrado, y el comienzo de los programas de estímulo al desempeño académico. A su vez, se trató de una política que incrementó las demandas laborales transformando el quehacer académico. Este artículo analiza cómo ha sido la diversificación de las funciones de los investigadores, el rol que han jugado los programas de incentivos, y la forma en la que han respondido tanto los investigadores como los integrantes de los espacios en los que se desempeñan. Éste es un trabajo que abona a los estudios organizacionales de la universidad ayudándonos a entender cómo cambia la naturaleza de las figuras, sus roles y funciones, conjuntamente con la universidad que los acoge. Ilustra algunas de las disyuntivas enfrentadas por los investigadores a partir de la implementación de políticas financieras impulsadas por mecanismos de evaluación, medición y recompensa.

Palabras clave: universidades, investigadores, desempeño académico, normatividad, estímulos económicos, Universidad Veracruzana, México.

\section{Elizabeth Ocampo Gómez}




\title{
O pesquisador fragmentado: conflitos e tensões derivadas da diversificação de sua carga laboral
}

\section{RESUMO}

A política de diversificação da carga instaurada nos anos noventa deu auge a ações encaminhadas à formação de redes e corpos académicos, a introdução do conceito de perfil de desempenho equilibrado, e o começo dos programas de estímulo ao desempenho académico. Por sua vez, se tratou de uma política que incrementou as demandas laborais transformando o trabalho acadêmico. Este artigo analisa como tem sido a diversificação das funções dos pesquisadores, o papel que têm tido os programas de incentivos, e a forma que têm respondido tanto os pesquisadores como os integrantes dos espaços nos que se desempenham. Este é um trabalho que auxilia os estudos organizacionais da universidade ajudando-os a entender como muda a natureza das figuras, seus papeis e funções, conjuntamente com a universidade que os acolhe. Ilustra algumas das disjuntivas enfrentadas pelos pesquisadores a partir da implementação de políticas financeiras impulsadas por mecanismos de avaliação, medição e recompensa.

Palavras chave: universidades, pesquisadores, desempenho acadêmico, regulamentação, estímulos económicos, Universidad Veracruzana, México.

The fragmented researcher: conflicts and tensions derived from the diversification of his workload

\begin{abstract}
The policy of diversification of the researchers' workload established during the 1990s gave rise to actions aimed at the formation of networks and academic bodies, the introduction of the concept of balanced performance profile, and the beginning of programs to stimulate academic performance. But it was also a policy that increased labor demands by transforming academic work. This article analyzes how the functions of researchers have diversified, the role played by incentive programs, and the way in which both researchers and members of the spaces in which they work have responded to the modifications. The article offers a contribution to university organizational studies by helping us to understand how the nature of the figures, their roles and functions change, together with the university that hosts them. It illustrates some of the dilemmas faced by researchers as a result of the implementation of financial policies driven by mechanisms of evaluation, measurement and reward.
\end{abstract}

Key words: universities, researchers, academic performance, regulations, economic incentives, Universidad Veracruzana, Mexico.

Recepción: 25/02/18. Aprobación: 11/04/19. 


\section{Introducción}

Este artículo examina los retos a los que se enfrentan los investigadores en una institución de educación superior (IES) que de manera creciente añade nuevas, variadas y adicionales responsabilidades a su carga laboral. Además, nos hemos propuesto explicar el rol que juega la estructura universitaria, la normatividad y los programas de incentivos para dirigir el desempeño de los investigadores en actividades que inicialmente no fueron especificadas para su tipo de contratación. Observamos que las crecientes demandas laborales hacia los investigadores han transformado su quehacer académico, sus estrategias para responder a las exigencias laborales, los objetivos que se plantean, su disponibilidad y consentimiento para participar en actividades, y los espacios institucionales donde acotan su atención. Reconocemos que muchas de estas transformaciones han sido abordadas en trabajos sobre educación superior (Acosta, 2006; Casillas, 1992; Galaz et al., 2008; Ibarra, 2003; Ibarra, 2010; Marugán y Cruces, 2013); aquí abonamos con un caso particular, el de la Universidad Veracruzana (UV), y con trabajo empírico desde el que recuperamos las percepciones de los investigadores adscritos a entidades de investigación. Siguiendo a Ibarra (2003: 338), vemos necesario atender el conocimiento detallado para entender los modos particulares de ser y de operar en la universidad. Nuestro análisis teórico se apoya en los trabajos desarrollados desde el campo de la contabilidad crítica (Miller y Rose, 1990), la gobernanza y el control a través de dispositivos de política pública (Foucault, 1978; Foucault, 1988; Rose, 1991; Shattock, 2013), el análisis de políticas educativas y estudios organizacionales (Casillas, 1992; Ibarra, 2003; Parsons 2012).

\section{Ubicando a la universidad de estudio}

La matrícula de la UV en educación formal es de 63369 estudiantes y cuenta con una planta de 6049 académicos (Universidad Veracruzana, 2017a: 61). Los estudios de posgrado son ofrecidos a través de 43 entidades de investigación, ${ }^{1}$ mismas que en su generalidad y por diseño institucional operan de manera separada de las licenciaturas. Cabe resaltar que hay facultades que actualmente ofrecen formación de posgrado, con un registro de 49 maestrías y 12 doctorados. $^{2}$ Históricamente, aunque las entidades de investigación hayan sido creadas en los primeros años de existencia de la UV, los programas de maestría comenzaron a operar 30 años después, ampliando su horizonte con la apertura de programas de doctorado a partir de este siglo. Actualmente la UV ofrece 124 de éstos, distribuidos en entidades de investigación y facultades, con una matrícula de 2007 estudiantes (UV, 2017a).

Antes, los investigadores habían estado adscritos mayoritariamente a los institutos y centros de investigación, pero en la actualidad, el porcentaje de investigadores en facultades e institutos se distribuye casi equitativamente. Participan en la universidad 549 investigadores, 462 con membresía del Sistema Nacional de Investigadores (SNI) y cuatro son integrantes del Sistema Nacional de Creadores de Arte (SNCA) del Instituto Nacional de Bellas Artes (INBA). De un total de 466 miembros del SNI y del SNCA, $60.47 \%$ está adscrito a entidades de investigación y $39.53 \%$ a facultades. En 2017, los investigadores impartieron 344 cursos de licenciatura. Respecto a la participación de los investigadores en la licenciatura, en este mismo año se desempeñaron como tutores de 1569 estudiantes de licenciatura, 612 de maestría y 431 de doctorado (UV, 2017a).

En una universidad donde las facultades son entidades académicas separadas de los institutos, los datos arriba citados, junto con el subsecuente análisis, evidencian que la tradicional separación de funciones para cada entidad se está diluyendo. Esto conlleva a explicar que a diferencia de muchas universidades

\footnotetext{
${ }^{1}$ Se incluyen centros e institutos de investigación.

${ }^{2}$ Datos recabados a partir de las páginas electrónicas de cada facultad al 30 de noviembre de 2017.
} 
del país donde no existe la división entre facultades e institutos, en la UV los investigadores mayoritariamente se desempeñaban en institutos, impartiendo docencia, tutoría de trayectorias académicas, enseñanza tutorial y asesoría de tesis en posgrado. Cabe aclarar que la UV tiene tres subcategorías de contratación dentro de la categoría de personal académico: académicos de carrera (que incluye a investigadores y docentes), técnicos académicos y docentes de asignatura (UV, 1994). ${ }^{3}$

Nuestra revisión de la normatividad muestra que a la par del crecimiento de las entidades de investigación y de la ampliación de la participación de investigadores en las licenciaturas, las políticas y la normatividad que rigen las funciones de los investigadores se han ido acotando, dirigiendo y controlando con mayor precisión y amplitud; añadiendo especificidad a los objetivos, la metodología y las políticas hacia éstos. A su vez, el trabajo empírico que realizamos da cuenta de cómo los académicos de carrera adscritos a institutos, en adelante referidos como investigadores, asumen responsabilidades en ámbitos internos y externos a su instituto y a su universidad, sin que existan, en muchos casos, los mecanismos de apoyo para que se lleve a cabo dicha flexibilidad.

\section{Fundamento teórico}

\section{Antecedentes de la diversificación de funciones}

En la segunda mitad de la década de los noventa se instauró una política de diversificación de la carga de trabajo dando auge a acciones encaminadas a la formación de redes y cuerpos académicos, la introducción del concepto de perfil de desempeño equilibrado, y el comienzo de los programas de estímulo al desempeño académico. Esto llevó a autores como
Ibarra (2003) y Gil y otros (1994) a vislumbrar el origen de un nuevo sujeto académico que se desempeñaría bajo un sistema de control, vigilancia y premiación o castigo. Otros reconocieron la figura del "académico integral" para referirse a la multiplicidad de funciones a desempeñar, a los cambios y a la complejidad de los nuevos roles (Galaz et al., 2008). En su revisión histórica, estos mismos autores asocian el crecimiento en la cobertura de las IES y el objetivo de justificar su pertinencia económica, con el desempeño de nuevas funciones en los académicos que más allá de actividades internas (investigación, docencia, tutoría, generación del conocimiento, gestión) incluyera trabajo y vinculación "con los sectores productivos de su entorno a través de actividades como asesoría, consultoría o investigación aplicada" (ibid.: 62).

Cabe resaltar que en la universidad que estudiamos ha jugado un papel fundamental el Modelo Educativo Integral y Flexible (MEIF), que rige la ruta curricular, administrativa y pedagógica adoptada por la UV desde 1999. ${ }^{4}$ A través del MEIF se introdujeron políticas federales marcadas para la educación superior tales como: el enfoque de formación integral, la implementación de un currículo flexible, las tutorías; todo lo anterior, perfilando la ruta hacia la diversificación de la carga académica.

\section{Diversificación: alcances, retos y tipos}

Las disyuntivas enfrentadas por los académicos para responder de manera estratégica a las demandas laborales en su empleo han sido ampliamente estudiadas. Por ejemplo, Galaz y colegas (2008) discuten sobre los dilemas para "decidir cómo invertir mejor su tiempo" en un sistema dónde los incentivos constituyen un "ingreso adicional muy significativo" (ibid.: 63). Otros, como Acosta (2006: 89), relatan cómo,

\footnotetext{
${ }^{3}$ La fecha de publicación 1994 no explica que este documento ha sufrido modificaciones continuas a sus artículos de esa fecha a la actualidad.

${ }^{4}$ A principios de la década de los noventa surgieron políticas nacionales que apostaban a la mejora y modernización de la educación superior (Casillas, 1992), el MEIF fue creado como respuesta a éstas.
} 
ante demandas a veces hasta contradictorias (trabajo individual vs. trabajo colectivo), los académicos son puestos en encrucijadas; y cómo la participación en programas de incentivos transforma y amplía sus quehaceres, enfoques, habilidades y destrezas. Las increpantes descripciones de Acosta hablan de una diversificación de actividades con un fuerte sentido pragmático (acumulación de puntos) y monetario donde lo académico queda desplazado.

Así también, el tema de la diversificación en la universidad es explicado por Marugán y Cruces (2013), pero desde la visión de diversificación de figuras laborales, identificando y problematizando la multiplicidad de categorías que ultimamente han surgido en la universidad. Ibarra (2003: 328) aporta elementos analíticos explicando cómo las universidades han vivido "una mayor parcelación de los espacios en los que cada quien es ubicado, siempre en función de la evaluación del rendimiento de acuerdo con una norma oficial previamente establecida". La crítica principal a un sistema que apuesta por tal diversificación es que "fragmenta al personal, lo divide y atomiza", lo pone a competir, dando prioridad a "trabajadores cualificados y versátiles, capaces de adaptarse" (Marugán y Cruces, 2013: 25).

A diferencia de nuestro trabajo, que busca explicar cómo el desempeño del académico es fragmentado por la creciente demanda de funciones para un solo puesto, estos autores enfatizan sobre las consecuencias de la diversificación de figuras y de espacios (oficinas) de control. No obstante, el nivel individual es abordado en sus críticas subrayando que la competencia entre los tipos de contrataciones produce precariedad laboral, cuya definición abarca "la dificultad de mantener trayectorias profesionales estables y continuas. Estas trayectorias están caracterizadas por la desregulación y por la ausencia de itinerarios profesionales rígidos" (ibid.: 28). Su descripción de precariedad cobra sentido cuando pensamos que los programas de sobresueldo otorgan niveles con vigencia temporal - siempre con la incertidumbre de poder renovarla- y cuyo sometimiento a sus indicadores implica cargas laborales que, como describiremos aquí, rebasan los límites de espacio y horario especificados para los puestos.

Shattock (2013: 225) es otro autor que confirma la inestabilidad del trabajo, derivada de condiciones demandantes, inestables y fragmentadas; él enfoca sus reflexiones a los resultados de la diversificación en la administración central universitaria. Con esto último, observamos cómo la fragmentación va desde la estructura organizacional compuesta por los espacios de gobierno para áreas específicas, hasta los individuos que son controlados desde la multiplicidad de oficinas que vigilan las multifacéticas fases de su desempeño.

\section{El resultado de los incentivos}

Las condiciones salariales de los académicos han sido estudiadas desde perspectivas y contextos heterogéneos. Los trabajos de Maldonado (2011) y de Altbach (2003) indican que sin los sobresueldos, los salarios de los académicos mexicanos son de los más bajos a nivel mundial. En esta tabla, mostramos de manera general las cantidades de los salarios base y las diferentes bolsas a las que pueden acceder los investigadores. Con los datos se sustenta el argumento del artículo que hace alusión a que los complementos salariales pueden más que duplicar el salario de los académicos de carrera. 
Tabla 1. Resumen de ingresos posibles por salarios y complementos correspondientes a los académicos de carrera

\begin{tabular}{|c|c|c|c|}
\hline Salario mensual académicos uv & sNI & PEDPA 2017-2019 & v040 \\
\hline $\begin{array}{c}\text { - Existen seis niveles: } \\
\text { Asociado A, B y C; } \\
\text { Titular A, B y C. } \\
\text { - El salario depende del nivel } \\
\text { y de la zona: } \\
\text { Asociado, de } \$ 13,964 \text { a } \$ 16,901^{*} \\
\text { Titular, de } \$ 27,768 \text { a } \$ 33,612^{*}\end{array}$ & $\begin{array}{l}-5 \text { Niveles } \\
\text { - Los incentivos van de } 3 \text { a } 14 \text { veces } \\
\text { el valor mensual de la UMA,** } \\
\text { de } \$ 7,350.72 \text { a } \$ 34,303.36\end{array}$ & $\begin{array}{c}-6 \text { Niveles } \\
\text { - Los incentivos van de } 1.5 \text { a } 8 \\
\text { salarios mínimo vigente }(\$ 80.04)^{* * *} \\
{ }^{* * * *} \text { De } \$ 2,401.2 \text { a } \$ 19,601.92\end{array}$ & $\begin{array}{c}\text { - Un pago anual } \\
\text { - El pago no podrá exceder } \\
\text { de } 14 \text { UMAS } \\
\text { **** } \$ 34,303.36 \text { para el nivel más } \\
\text { alto. }\end{array}$ \\
\hline
\end{tabular}

Fuente: Elaboración propia con datos de la uV (2018b; 2017c), el INEGI (2019), el CONACYT (2017), FESAPAUV (2018).

SNI: Sistema Nacional de Investigadores; PEDPA: Programa de Estímulos al Desempeño del Personal Académico UV; U040: Programa de Carrera Docente.

* Datos obtenidos del Sindicato Estatal de Personal Académico de la Universidad Veracruzana (FESAPAUV), 2018.

** UMA: Unidad de Medida Actualizada, $\$ 80.60$

*** El salario mínimo está calculado con datos del 2017, en tanto que el PEDPA estudiado abarca el bienio 2017-2019.

**** Cálculo aproximado de acuerdo con la información disponible.

Distintas reflexiones surgen cuando pensamos en la proporción tan alta de los incentivos en relación con los salarios. Primero, no se crean derechos sobre los incentivos, sino que son concesiones sujetas a renovación que, de no cumplir con los requisitos estipulados para cada uno, se justifica removerlos. Esto produce lo que explicamos anteriormente como precariedad, pero también insatisfacción laboral, estudiada por Padilla y otros (2013: 15), quienes observan que la baja satisfacción laboral de los académicos adscritos a centros de investigación podría estar relacionada con la poca estabilidad laboral y la sujeción a "estándares altos de exigencia y evaluación”. Segundo, los sobresueldos son para personal en activo, por lo que preocupa lo que sucede con los jubilados que se retiran con sueldos de bajo poder adquisitivo. Tercero, gozar del sobresueldo oculta, al menos temporalmente, la precariedad del sueldo base, creando una ilusión de suficiencia. Cuarto, la incertidumbre de mantenerlos o perderlos produce sujeción a las exigencias o, en palabras de Foucault (1988), disposición del beneficiario a cumplir con las demandas institucionales. De tal forma que los beneficiarios transforman sus conductas, acciones, destrezas, como lo hemos venido explicando y como evidenciaremos con el trabajo empírico realizado para este estudio. Sobre este asunto, Ibarra (2003: 306) reflexiona:
al establecerse tales índices e indicadores, privile- giando ciertas acciones en lugar de otras, se afectan de manera directa la naturaleza, el contenido y la organización del trabajo universitario, funcionando como medios < naturales $>$ y $<$ objetivos $>$ de jerar- quización, clasificación y diferenciación; la precisión y la transparencia son sus placas predilectas de legi- timación, el error su máximo enemigo.

Por su parte, Miller y Rose (1990: 8) hablan de la evaluación y los procedimientos de examinación como tecnologías de gobierno cuyo uso tiene el potencial de moldear conductas a distancia. Rose (1991: 676) explica, "los eventos deben ser inscritos en formularios estandarizados, las inscripciones transportadas a lo largo y ancho y acumuladas en una ubicación central, donde se puedan juntar, 
comparar, compilar y calcular". Abonando a la discusión sobre el rendimiento de cuentas o los reportes del desempeño, Ocampo y Neu (2008: 43) explican que "la contabilidad facilita los actos de dividir/disciplinar y dividir/recompensar ayudando a construir 'un campo de comparación' que permita a uno 'diferenciar individuos entre sí”". Aquí postulamos que la participación en programas de incentivos genera mediciones numéricas que se constituyen en material contable; números que justifican intervenciones.

\section{Aproximación metodológica}

El reporte presentado aquí forma parte de una investigación más amplia en la que exploramos aspectos generales del modelo educativo de la UV. En torno a los investigadores nos interesaba conocer el origen, los alcances y dilemas de la diversificación de la carga laboral. Este interés surgió en parte porque al estudiar temas referentes a la licenciatura encontramos tensiones relacionadas con el desempeño docente de los investigadores en el nivel de la licenciatura y derivadas de una estructura donde se encuentran separadas las facultades de los institutos. Sin embargo, en la práctica y de manera creciente, los investigadores adscritos a institutos ahora buscan participar en las licenciaturas, en parte debido a las políticas públicas que fomentan un desempeño en diferentes niveles. Con base en el tipo de interrogantes planteadas hicimos trabajo documental y trabajo de campo sobre el tema. En el análisis documental incluimos los documentos normativos de la UV (el Estatuto del Personal Académico, la Ley Orgánica), así como las convocatorias junto con los lineamientos y la descripción de indicadores que se consideran en las evaluaciones de los programas de sobresueldo en los que participan los investigadores. El trabajo empírico incluyó la aplicación de un cuestionario con seis preguntas abiertas (Tarrés et al., 2013). Éstas fueron planteadas en relación con su participación en las licenciaturas de la siguiente forma:
- ¿Qué conoce y cómo entiende su rol en el desarrollo de habilidades investigativas?

- ¿Por qué participan o desean participar?

- ¿Cómo participan?

- ¿Qué problemáticas enfrentan?

- ¿Qué prácticas exitosas han desarrollado o identifica que se desarrollan?

- ¿Qué proponen?

El cuestionario fue enviado a través de los directores de todas las entidades de investigación. Solamente los directores de 10 institutos, siete centros de investigación y un laboratorio difundieron la invitación entre los integrantes de sus dependencias. Recibimos 88 cuestionarios contestados del sondeo realizado a las entidades arriba mencionadas. Tras este ejercicio, convocamos a directores e investigadores a participar en cuatro grupos focales donde se incorporaron tres entidades más. Los grupos fueron estructurados de manera interdisciplinar para conocer cómo participaban en las licenciaturas y cuáles eran sus propuestas para mejorar su inserción en éstas. Esta agrupación interdisciplinar también se debió al deseo de que se compartieran buenas prácticas que ampliaran el imaginario de quienes enfrentan problemas similares. Usamos grupos focales por sus múltiples alcances: la externalización de ideas individuales en un marco colectivo, el poder que tiene el testimonio grupal, su potencial pedagógico y dialéctico; y en términos más prácticos, por la rapidez con la que se genera información con varios participantes (Kamberelis et al., 2011). Cada uno de los grupos respectivos, compuestos por un promedio de 11 participantes, trabajó sobre 10 preguntas guía. Posterior a esto se integraron en un grupo general otros investigadores interesados y algunas autoridades universitarias con quienes se realizó una plenaria donde se socializaron los resultados de cada grupo, se intercambiaron ideas y reforzaron algunos argumentos. Toda esta información fue registrada mediante formatos-guía donde incluimos las preguntas arriba 
mencionadas. Los grupos nos entregaron tanto sus formatos, como archivos PowerPoint que elaboraron para compartir sus reflexiones en la plenaria. Adicionalmente tomamos notas de campo y videograbamos las plenarias.

Una vez integrados todos los datos, procedimos a examinarlos mediante una metodología cualitativa de análisis de contenido (Berg, 2001). Trabajamos con el software ATLAS.ti integrando una codificación con base en la estructura de las preguntas de la recolección de datos. La mayoría de los códigos utilizados fueron previamente establecidos y definidos con base en los objetivos de conocer por qué y cómo participan los investigadores en el nivel de licenciatura. Éstos fueron a su vez fundamentados en la teoría organizacional y de análisis de políticas educativas. Un número menor de códigos surgió in-vivo a partir de la lectura y procesamiento de los datos y de la identificación de temáticas que no se habían previsto.

\section{El rol de los programas de incentivos}

Los cuatros principales programas de incentivos en los que pueden participar los investigadores son: el Sistema Nacional de Investigadores (SNI), el Programa para el Desarrollo Profesional Docente (PRODEP), el Programa de Estímulos al Desempeño del Personal Académico (PEDPA) UV, y más recientemente el Programa de Carrera Docente U040.

Queremos destacar algunos elementos que refuerzan la citada diversificación de la carga laboral desde estos programas. Primero, la multiplicación de estas bolsas y los montos a los que se puede acceder a través de éstas hace alusión al referido control laboral que se puede ejercer sobre los académicos (Galaz et al., 2008; Ibarra, 2003; Marugán y Cruces, 2013). Lo anterior, particularmente considerando que, como ya señalamos, estas bolsas pueden duplicar o casi triplicar el ingreso en proporción al sueldo base.
El SNI, por ejemplo, privilegia las actividades de investigación y formación de investigadores evidenciadas a través de la producción científica y tecnológica y mediante la dirección de tesis culminadas. La asignación de un grado SNI otorga remuneración económica pero también reconocimiento simbólico (Acosta, 2014; Ocampo y Rueda, 2015).

Del PRODEP subrayamos su Reconocimiento a Profesores de Tiempo Completo a Perfil Deseable ${ }^{5}$ porque obtenerlo significa demostrar un desempeño diversificado, y porque, aunque dicho reconocimiento no representa un sobresueldo, es un requisito para el acceso de los académicos a la obtención individual de recursos extraordinarios externos a la UV, y requisito para participar en otros programas de incentivos.

Aunado a esto, en los últimos años la Universidad ha venido otorgando un estímulo económico adicional pagado en una sola exibición (UV, 2017c) a sus beneficiarios. El reconocimiento a perfil deseable requiere cuatro actividades que los aspirantes deben realizar, y aunque no todas son consideradas sustantivas, el académico debe demostrar, según los criterios establecidos, la totalidad de las mismas (PROMEP, s/f: 29).

Por otro lado está el incentivo de la UV conocido como el Programa de Estímulos al Desempeño del Personal Académico (PEDPA). Éste valora los mismos rubros que los anteriores, con mínimos a cubrir considerados como las actividades inherentes al puesto. En su convocatoria se especifica como uno de los objetivos: "Reconocer y estimular a los académicos [que realizan] actividades integrales extraordinarias a las que obligadamente debe desempeñar" (UV, 2017c). Así, se valora el desempeño en los siguientes rubros generales: calidad, permanencia, dedicación, docencia, generación y aplicación del conocimiento y gestión académica.

\footnotetext{
${ }^{5}$ Además de reconocimiento al Perfil Deseable, PRODEP emite convocatorias para: Apoyo a la Incorporación de Nuevos Profesores de Tiempo Completo; Apoyo a la Reincorporación de exbecarios. PROMEP; Apoyo a Profesores de Tiempo Completo con Perfil Deseable; Apoyo para estudios de Posgrados de Alta Calidad, y Apoyo para el Fortalecimiento de los Cuerpos Académicos.
} 
Existe también otro incentivo de más reciente creación conocido como el Programa de Carrera Docente (Fondo extraordinario) (UV, 2018a). Éste solicita que se cumplan al menos 5 de 10, indicadores especificando como requisito para participar contar con Perfil Deseable de PRODEP y haber obtenido un nivel en la última convocatoria del PEDPA. Destaca el número de indicadores que valoran las actividades en licenciaturas, incluyendo un indicador que asocia la membresía al SNI con la impartición de cursos en licenciatura.

Es de resaltar que la inclusión de los programas de incentivos a la productividad de los académicos en la UV da cuenta de la focalización en cuanto a política institucional para promover, a través del reconocimiento y estímulo económico, que los académicos cubran otras funciones además de las que son inherentes y sustantivas en correspondencia con su tipo de contratación. Esto se ha venido reforzando a través de los años mediante la clarificación de definiciones de las categorías, indicadores y condiciones o criterios para participar en estos programas de sobresueldo.

\section{La perspectiva de los participantes}

Aquí presentamos los resultados de nuestra indagación sobre los motivos de los investigadores para participar en la licenciatura. La mayoría de las citas de este apartado provienen de la información obtenida a través de los grupos focales y cuestionarios arriba referidos. Nuestro análisis expone tres temáticas generales (elementos en juego para participar, retos enfrentados e interacciones intrainstitucionales) que se derivaron de nuestra lectura de los documentos generados a partir de los cuestionarios contestados y los grupos focales. Las temáticas están alineadas con nuestro diseño de los instrumentos, pero también contemplan categorías más específicas que emergieron a partir de los datos, explicadas a continuación.

\section{Elementos en juego para participar}

En este estudio proveemos datos sobre algunos de los elementos que juegan un rol importante en la decisión para participar dentro de los que se incluye la participación por conveniencia o necesidad, por requisito institucional o por vocación.

Dentro de la participación por conveniencia o necesidad recuperamos ideas que aluden al deseo de permanecer o mejorar su situación laboral. Por ejemplo, hicieron mención del reclutamiento y preparación de estudiantes para el posgrado (Investigaciones Psicológicas 8: 1_248; Ciencias Básicas 12: 3_162; SARA ${ }^{6}$ 10: 2_122). Este interés está vinculado con la identificación de jóvenes con potencial para la investigación. Los jóvenes reciben formación pero a su vez contribuyen a indicadores medibles en beneficio de los investigadores, tales como la formación de capital humano, que es uno de los criterios considerados en el SNI.

Otros hicieron alusión abierta a participar porque este tipo de espacio les abre más posibilidades de obtener incentivos. Por ejemplo, uno relata, "He participado porque es un requisito para productividad" (Ciencias Básicas 4: 2_217); a lo que se suman otros investigadores $\left(\mathrm{CECDA}^{7}\right.$ 1: 2_367).

Además, encontramos quienes hicieron alusión a razones derivadas de las funciones que la universidad ha hecho inherentes a su puesto (Inbioteca 7: 2_436; CEnDIUH $^{8}$ 1: 2_386; Ciencias Básicas 5: 2_211; Inbioteca 4: 2_420; $\operatorname{IIESES}^{9} 1: 2 \_9$ ). Por ejemplo, la mayoría explicó que la diversificación es una expectativa de la institución, misma que es percibida por los investigadores como una buena práctica (Ciencias de la Tierra 1:4_241; Investigaciones Psicológicas 8: 5_252; SARA 6: 5_89; Ciencias Básicas 10: 5_175). Algunos

\footnotetext{
${ }^{6}$ Servicios de Apoyo en Resolución Analítica.

${ }^{7}$ Centro de Estudios, Creación y Documentación de las Artes.

${ }^{8}$ Centro de Desarrollo Humano e Integral de los Universitarios.

${ }^{9}$ Instituto de Investigación y Estudios Superiores Económicos y Sociales.
} 
afirmaron tener carga diversificada mediante su participación en dos o más actividades en los programas educativos de las licenciaturas, lo que implica realizar docencia en asignaturas del plan de estudios correspondiente o en asignaturas de libre elección, tutorías académicas, tutorías para la investigación, para la apreciación artística, prácticas profesionales, proyectos de investigación, experiencia recepcional, servicio social, participar en academias de facultades y en la elaboración de los planes de estudio. Todas estas actividades respaldadas por la política de diversificación forman parte de la referida fragmentación a la que son sometidos los investigadores. Como ejemplo de todo lo que pueden hacer, uno refiere: "como docente creando nuevos programas en cada experiencia; como tutor, participando con el estudiante en su proceso formativo; como director o jurado de tesis haciéndome copartícipe de la experiencia" (Lingüístico Literarias, 3: 2_220); a lo que hacen eco otros (SARA 8: 5_80; Ciencias de la Salud 5: 1_316; SARA 6: 3_87). Al respecto, Ruiz y Aguilar (2017: 39) proveen un análisis de este tipo de requerimientos en su libro sobre competencias del profesorado universitario, remarcando que "la universidad está viviendo uno de los momentos de mayor transformación de su historia, que conlleva nuevos requerimientos y nuevas exigencias para el profesorado".

De manera paralela encontramos afirmaciones que hicieron referencia a una participación que responde a los fines planteados por la institución, mencionando querer incidir en el objetivo del MEIF en relación con la formación integral de los estudiantes (Ciencias de la Salud 2: 3_341). Es decir, se percibe que con el acercamiento a los investigadores, muchos estudiantes logran extender su formación hacia otras disciplinas y hacia la investigación (SARA 6: 5_89; Ciencias Biomédicas, 2: 10_57; Ciencias Biomédicas 5: 3_30). Finalmente, encontramos respuestas que denotan un sentido de responsabilidad social y vocación en tanto que indican participar por gusto o retribución a la sociedad (SARA 1: 2_116).

\section{Retos enfrentados al participar}

$\mathrm{Al}$ indagar sobre los desafíos enfrentados descubrimos que uno de los principales emana de la separación estructural entre facultades e institutos que prevalece en la universidad. Bajo tal separación entran en juego varios intereses personales y de grupo. Por ejemplo, al investigador le conviene o interesa (como ya describimos arriba) participar en las facultades a través de la licenciatura; los casos donde así sucede suelen despertar preocupaciones en tanto que se remueve el status quo del desempeño de quienes están adscritos a éstas, por ejemplo, uno refiere "Es lamentable ver que aún se mantiene esa visión anticuada de competencia entre los profesores y los investigadores cuando podríamos ser colaboradores en beneficio de todos" (Inbioteca 5: 3_424).

Por otro lado, dar cabida amplia a la participación de investigadores en la licenciatura puede poner en riesgo la disponibilidad de plazas a convocar en las facultades. Con todos los motivos para desempeñarse en las licenciaturas, los investigadores manifiestan tener más disponibilidad de la que quizás muchos directivos y docentes de las facultades están dispuestos a aceptar. Lo anterior porque es mediante la contratación de nuevo personal, en ocasiones con filias previamente establecidas, que se pueden crear fidelidades políticas al interior de las facultades. Por lo tanto, la licenciatura se convierte en un terreno de contención, de vigilancia y estrategias cautelosas cuando se desea participar (Ciencias de la Salud 4: 3_325; Ciencias Básicas 8: 3_197).

Otra barrera que complejiza la participación de los investigadores en la licenciatura se encuentra en la forma en la que es organizada la oferta de las asignaturas. Considerando que se trata de oferta casi siempre flexible, los calendarios no facilitan que los investigadores armonicen sus espacios, tiempos y los compromisos de su función sustantiva, como explica uno: "Si la experiencia educativa se cruza con mi horario en mi instituto, simplemente no puedo impartirla (Investigaciones Psicológicas 6: 3_263). 
Otros más refieren la misma problemática (Investigaciones Psicológicas 8: 3_250; Salud Pública 2: 3_152; MICRONA ${ }^{10}$ 1: 4_380; Ciencias Biomédicas 2: 9_56; Grupo Focal 3: 6). De manera similar se hizo alusión al desfase de materias en tanto que el calendario de posgrado es diferente al de licenciatura.

En la descripción de estos retos entran en juego otros elementos como la disponibilidad de los estudiantes para tomar tutorías para la investigación, hacer su servicio social con algún investigador, u otro tipo de colaboración entre investigadores y estudiantes de licenciatura. En muchos casos, los horarios de clase distribuidos por todo el día impiden que los estudiantes realicen actividades con investigadores. Un investigador describe lo anterior con claridad:

las distancias también influyen, ya que al tener una, dos o tres horas libres y quieren trabajar en su proyecto de investigación, requieren de cerca de dos horas (ida y vuelta) para el traslado de la facultad al ICB y el regreso. De tal manera que en una hora no hacen nada en el laboratorio. Todo ello derivado de la falta de infraestructura de la facultad y, por lo mismo, de la inflexibilidad del MEIF en la facultad (Ciencias Básicas 7: 2_201).

Esta misma observación es reiterada en la participación de otros investigadores (Cerebrales 1: 4_314; Salud Pública 6: 4_133).

Otro tema importante, y que tiene que ver con la normatividad, es que la Universidad espera la diversificación de las funciones -aun cuando ésta no ha sido reglamentada, ni se han establecido criterios administrativos y normativos para establecer cómo funciona (CECDA 2: 4_363; IIESES 1: 4_11; SARA 8: 4_79; Ciencias Básicas 5: 4_213; Grupo Focal 3: 5). Lo notable en torno a dicha diversificación es que a pesar de la floja articulación normativa, los investigadores acceden a aumentar su carga laboral desempeñándose en funciones no diseñadas para su cargo y en muchas ocasiones no pagadas o remuneradas vía estímulos (Ciencias de la Salud 3: 5_336; SARA 9: 6_73; Ciencias de la Salud 2: 6_344). Este inédito acto de altruismo laboral es lo que nos hemos propuesto explicar aquí. Miller y Rose (1990) hacen un recuento histórico de todas las aproximaciones teóricas al estudio de los mecanismos para regular la vida productiva; siempre apuntando cómo las instituciones implementan estrategias para incrementar la producción. Evidenciamos en este estudio la plétora de estrategias implementadas desde la UV para que los investigadores tengan la disposición para realizar actividades "integrales extraordinarias a las que obligadamente debe desempeñar", como señala el reglamento del PEDPA. Esto es referido por algunos como la minimización de las resistencias o los mecanismos implementados para moldear conductas (Miller y Rose, 1990; Marugán y Cruces, 2013).

\section{Interacción intra-institucional (obstáculos, ambiente desfavorable)}

Como parte de este estudio diseñamos algunas preguntas para indagar sobre las percepciones ${ }^{11}$ de los investigadores en torno a su interacción en las facultades. Dentro de esta exploración encontramos que un número significativo de investigadores perciben que su trabajo en la licenciatura es desvalorado, el grupo focal 4 propone que "tiene que haber apertura valorativa por ambas partes, respeto mutuo" (Grupo Focal 4: 2). Cuando describen sus estrategias para incursionar en dicho nivel educativo mencionan que algunos directivos les hacen parecer que permitirles el acceso es un favor:

en nuestro centro no contamos siquiera con un salón para impartir las asignaturas y debemos recorrer

\footnotetext{
${ }^{10}$ Micro y Nanotecnología.

${ }^{11}$ Por percepción entendemos los sentimientos, las impresiones recibidas mediante los sentidos y las intuiciones; todas éstas enmarcadas por experiencias personales y profesionales (Vargas, 1994).
} 
diversas dependencias para ver si pueden hacernos el favor de prestarlo, como si se tratara de otra universidad. Inclusive nos cobran por hacer la limpieza!!!! (Ciencias Biomédicas 1: 9_67).

Este tipo de explicación es respaldada por otros (SARA 5: 4_94; SARA 2: 3_113; Grupo Focal 3: 4; Grupo Focal 1: 2). De esta forma la contribución queda, o al menos así se percibe, poco apreciada. También describen que otros colegas docentes se sienten desplazados por el interés de los investigadores de aumentar sus horas de clase o de ocupar una plaza de asignatura. Esto es porque al asignar las horas en licenciaturas a personal adscrito a posgrado, se vuelven injustificadas las nuevas contrataciones.

Algunos investigadores compartieron que la universidad podría ser estratégica para aprovechar su disponibilidad de trabajar en la licenciatura, evitando así la contratación de nuevo personal o el uso del banco de horas de las facultades en tanto que ellos pueden y desean cubrir asignaturas como parte de su carga, pero además, para participar en incentivos como el U040 o el PEDPA, descritos arriba. El grupo focal 4 sugirió:

Convocar perfiles más abiertos para las asignaturas de licenciatura para que realmente sean convocatorias abiertas en las que puedan participar los investigadores [...] Previamente a la asignación de materias vacantes por Consejo Técnico, dar a conocer a los institutos y centros las materias y sus programas para captar en primera instancia el complemento de carga de los investigadores que cumplan con el perfil (Grupo Focal 4: 2).

Esta opinión es respaldada por otros (Ciencias de la Salud 3: 5_336; SARA 8: 4_79; Grupo Focal 2: 7). Este tipo de propuesta fue, en buena medida, lo que despertó nuestro interés para realizar la presente investigación. La redacción de este artículo fue motivada tras leer y escuchar en el material recabado, lo que interpretamos como el deseo de trabajar más a allá de lo que marca la legislación para su tipo de contratación. Como investigadoras quisimos conocer qué propiciaba esto. Cabe aclarar que las expresiones de anhelo de trabajar más y de ahorrarle dinero a la universidad se enmarcaron en los eventos financieros suscitados en el 2016, año en el que se hizo pública la ausencia de subsidio estatal y el déficit, de más de 2000 millones de pesos, en el que se encontraba la Universidad Veracruzana (UV, 2016: 128-131). No obstante, indagando a mayor profundidad, descubrimos que junto al deseo de contribuir al precario estado financiero en el que se encontraba la Universidad, había un interés alentado por los incentivos. Por tanto, este tipo de aseveración entreteje benevolencia y generosidad con el poder del complemento a un salario que de otra forma sería demasiado bajo (Altbach, 2003; Maldonado, 2011; Vázquez, 2012).

Con esto se ejemplifica cómo el sujeto se hace maleable a partir de la implementación de mecanismos financieros que van desde un salario rudimentario, hasta la puesta en marcha de incentivos cuyo volumen es igualmente proporcional al grado de coerción del sistema (Acosta, 2014; Baldwing y Krostseng, 1985).

De manera similar, expresaron un deseo por trabajar juntamente con los profesores de las facultades correspondientes. Tal deseo no siempre es posible por variadas situaciones, dentro de las que se hace presente la referida contienda territorial, en tanto que las divisiones organizacionales juegan un papel en la conformación de territorios políticos dentro de la institución educativa (Agnew, 2000; Gottmann, 1975; Raffestin, 1993) donde la facultad y sus integrantes se posicionan en un frente diferente al de la investigación y los investigadores, buscando proteger el espacio dentro del que son los actores con mayores privilegios y control.

\section{Conclusiones}

Explicamos a través de datos y de trabajo empírico cómo las funciones que actualmente desempeñan los investigadores trascienden en mucho las tareas 
acotadas a la investigación. Esto ha tensionado la identidad del investigador y la delimitación entre los espacios de su desempeño. Utilizamos en el título de este artículo el adjetivo "fragmentado" para referir a un investigador que desempeña funciones diversas, no porque postulemos que su desempeño deba ser unidireccional; creemos que su participación en diferentes ámbitos de la universidad puede enriquecer los procesos y los resultados. No obstante, el argumento aquí expuesto es que las políticas universitarias no favorecen su participación en las áreas donde ellos pueden destacar, sino que inducen la participación en todos los ámbitos marcados por los programas de incentivos, contribuyendo así a la descrita fragmentación.

Describimos las características y percepciones de tal fragmentación observando una asimetría entre la expectativa laboral y las condiciones para su materialización. Cabe mencionar que, aunque se hayan precisado los indicadores y las condiciones para tomarlos en cuenta, aún se identifica que coexisten elementos y tareas de escasa o nula evaluación, zonas no cuantificadas y por tanto no valoradas. Tal situación conlleva a mediciones que no necesariamente reflejan o encaminan al logro de objetivos institucionales sino, en muchas ocasiones, a simulaciones; es decir, fomentando que quienes participan en los programas de incentivos concentren el beneficio en sí mismos y no en las tareas sustantivas de la institución.

Contrastando estos resultados con las percepciones de los investigadores encontramos una clara disposición para participar en una variedad de actividades, a pesar de tratarse de actividades que incrementan o incluso pueden duplicar su trabajo, que se realizan en condiciones desfavorables, bajo una estructura organizacional que no lo propicia ni lo facilita, y bajo dinámicas institucionales de contienda territorial. Juegan un papel concomitante tanto el marco normativo como los programas de incentivos en tal avenencia laboral. A la luz de la teoría encontramos que en un contexto laboral de bajos sueldos, el incentivo económico cumple una función de mecanismo de gobierno que direcciona el desempeño sometiendo al trabajador a exigencias que sobrepasan lo especificado para su puesto (Marugán y Cruces, 2013). Por tanto, logramos no sólo corroborar el papel que juega la normatividad en la ampliación de funciones y el rol de los incentivos, sino anclar varios problemas expresados por parte de los investigadores en torno a las demandas y condiciones laborales. También encontramos un rompimiento de funciones especificadas entre figuras porque, aunque los puestos son diferentes (docentes, investigadores, técnicos académicos), las expectativas en torno a las actividades que desempeñan contemplan que asuman tareas en todos los ámbitos en los que se asigna puntaje en los programas de incentivos. Pareciera que lo único que diferencia los puestos es la formación, la categoría y el sueldo de quienes los ocupan, más no las actividades, en tanto que se invaden espacios de acción.

Cerramos subrayando que esta investigación abre vertientes para estudios posteriores. Algunas de éstas son: analizar qué sucede a nivel personal con los académicos que se desempeñan en una estructura progresivamente demandante; explorar cómo transitan en la institución académicos que han sido marginados o que han decidido no participar en los programas de incentivos; discutir qué se pierde a nivel institucional y profesional cuando los investigadores se involucran en actividades crecientemente diversificadas, cómo se afecta la naturaleza del trabajo universitario; explorar cómo se ve impactada la licenciatura con la participación de los investigadores; debatir en qué medida las políticas de diversificación contribuyen a la homogenización de perfiles, a la desvaloración de vocaciones individuales y a la pérdida de la autonomía; investigar si la fragmentación de funciones deviene en un desempeño superficial o de simulación; realizar un estudio genealógico de la normatividad para conocer cómo se han ido configurando los cambios que han dado lugar al actual panorama laboral que hemos descrito en este trabajo. 


\section{Referencias}

Acosta, Adrián (2014), "El poder de los incentivos", Campus Milenio, núm. 549, <http://www. campusmilenio.com.mx/index.php/template/ opinion/estacion-de-paso/item/1322-el-poder-de-losestimulos> [Consulta: marzo de 2014].

Acosta, Adrián (2006), "Señales cruzadas: una interpretación sobre las políticas de formación de cuerpos académicos en México", Revista de la Educación Superior, vol. XXXV (3), núm. 139, pp. 81-92.

Agnew, John (2000), "Global Political Geography beyond Geopolitics", International Studies Review, vol. 2, núm. 1, Oxford University Press, pp. 91-99, <https://doi. org/10.1111/1521-9488.00185>.

Altbach, Philip (2003), "Evaluar y recompensar a profesores: el estilo mexicano", Campus Milenio, núm. 27, $<$ http://www.ses.unam.mx/publicaciones/articulos.ph p?proceso $=$ visualiza\&idart $=1158>\quad[$ Consulta: marzo de 2014].

Baldwing, Roger y Marsha Krostseng (1985), "Incentives in the academy: Issues and options", Incentives for faculty vitality. New directions for Higher Education, núm. 51, pp. 50-20 [DOI: 10.1002/he.36919855103].

Berg, Bruce (2001), Qualitative research methods for the social sciences, Boston, Allyn and Bacon.

Casillas, Juan (1992), "Dos puntos de vista sobre la Educación Superior, Los compromisos de las Instituciones de Educación Superior en México", Universidad Futura, vol. 4, núm. 10, pp. 34-48.

CONACyT (2017), "Acuerdo por el que se emite el nuevo Reglamento del Sistema Nacional de Investigadores", Cap. XI, arts. 30-33, México, <https://www.conacyt. gob.mx/index.php/el-conacyt/sistema-nacional-deinvestigadores/marco-legal/reglamento-sni/13493reglamento-sni/file> [Consulta: noviembre de 2017].

FESAPAUV (2018), "Contrato Colectivo de Trabajo del Personal Académico", Veracruz, FESAPAUV/Universidad Veracruzana 2018-2020, <http://www.fesapauvxalapa. org/archivos/ContratoColectivoFESAPAUV.pdf> [Consulta: marzo de 2019].
Foucault, Michel (1988), "El sujeto y el poder", Revista Mexicana de Sociología, vol. 50, núm. 3, pp. 3-20, <DOI: $10.2307 / 3540551>$.

Foucault, Michel (1978), "La gubernamentalidad", en Robert Caste et al., Espacios de poder, España, La Piqueta, pp 9-26.

Galaz, Jesús, Laura Padilla, Manuel Gil y Juan Sevilla (2008), "Los dilemas del profesorado en la educación superior mexicana", Calidad en la Educación, núm. 28, <https://calidadenlaeducacion.cl/index.php/rce/ article/view/202/207> [Consulta: febrero de 2019].

Gil, Manuel, Rocío Grediaga, Lilia Pérez, Norma Rondero y Miguel Casillas (1994), Los rasgos de la diversidad: un estudio sobre los académicos mexicanos, México, UAM.

Gottmann, Jean (1975), "The evolution of the concept of territory", Social Scienc Information, vol. 14, núm. 3, Maison des Sciences de l'Homme, <https://doi. org/10.1177/053901847501400302> [Consulta: marzo de 2018].

Ibarra, Eduardo (2010), "Exigencias de organización y de gestión de las universidades públicas mexicanas: de su pasado político a sus mercados presentes", en Daniel Cazés et al. (2010), Las Universidades públicas mexicanas en el año 2030: examinando presentes, imaginando futuros, México, UNAM, Centro de Investigaciones Interdisciplinarias en Ciencias y Humanidades, pp. 55-92.

Ibarra, Eduardo (2003), La universidad en México hoy: gubernamentalidad y modernización, México, UNAM/ UAM-I/ANUIES [Col. Posgrado, 16].

Instituto Nacional de Estadística y Geografía (INEGI) (2019), INEGI-Precios-UMA, <https://www.inegi.org. mx.temas/uma> [Consulta: marzo de 2019].

Kamberelis, George y Greg Dimitriadis (2011), "Focus groups: contingent articulations of pedagogy, politics, and inquiry", en N. K. Denzin y Y. S. Lincoln (eds), The SAGE Handbook of Qualitative Research, EUA, SAGE Publications, pp. 545-562. 
Maldonado, Alma (2011), "Salarios de académicos de instituciones de educación superior en México comparados con 30 países", en XI Congreso Nacional de Investigación Educativa, México, COMIE, <http://www. comie.org.mx/congreso/memoriaelectronica/v1l/ docs/area_16/2395.pdf> [Consulta: diciembre de 2017].

Marugán, Begoña y Jesús Cruces (2013), "Fragmentación y precariedad en la universidad", Sociología del Trabajo, núm. 78, pp. 10-34.

Miller, Petery Nikolas Rose(1990), "Governing Economic Life", Economy and Society, vol.1, núm. 19, pp. 1-31.

Ocampo, Elizabeth y Dean Neu (2008), Doing missionary work: The World Bank and the diffusion of financial practices, New Brunswick, Fernwood Publisher.

Ocampo, E. y J. Rueda (2015), "El Sistema Nacional de Investigadores en la Universidad Veracruzana: análisis exploratorio de cómo se experimenta el reconocimiento académico", Revista Interamericana de Educación de Adultos, núm. 37 (1), pp. 64-85.

Padilla, Laura, Leonardo Jiménez y María Ramírez (2013), "La satisfacción laboral en el personal académico y su relación con la intención de abandonar la profesión”, Perfiles Educativos, vol. XXXV, núm. Especial.

Parsons, Wayne (2012), Politicas públicas. Una introducción a la teoría y la práctica del análisis de las políticas públicas, México, FLACSO.

PROMEP (s/f), "Informe ejecutivo", México, <http:// www.dgesu.ses.sep.gob.mx/documentos/DSA\%20 gobmx/Informe\%20Ejecutivo\%20Promep.pdf> [Consulta: diciembre de 2017].

Raffestin, Claude (1993), Por uma geografia do poder, São Paulo, Atica.

Rose, Nicholas (1991), "Governing by numbers: figuring out democracy", Accounting Organizations and Society, núm. 7, vol. 16, pp. 673-692.

Ruiz-Corbella, Marta y Marlene Aguilar-Feijoo (2017), "Competencias del profesorado universitario: elaboración y validación de un cuestionario de autoevaluación", Revista Iberoamericana de Educación Superior, vol. VIII, núm. 21, pp. 38-65.

Shattock, Michael (2013), "University governance, leadership and management in a decade of diversification and uncertainty", Higher Education Quarterly, vol. 67, núm. 3, pp. 217-233.

Tarrés, María-Luisa (coord.) (2013), Observar, escuchar y comprender: sobre la tradición cualitativa en la investigación social, México, FLACSO.

Universidad Veracruzana (2018a), "Convocatoria para participar en el programa de carrera docente U040", Veracruz, <https://www.uv.mx/dgdaie/ files/2018/04/CONVOGATORIA-U040-2018. pdf $>$ [Consulta: febrero de 2019].

Universidad Veracruzana (2018b), “Tabulador del personal académico", Veracruz, UV-Dirección General de Recursos Humanos, <https://www. uv.mx/ingenieriacivil/files/2018/03/ResultadoNegociaciones_2018.pdf $>$ [Consulta: febrero de 2019].

Universidad Veracruzana (2017a), IV Informe de Actividades 2016-2017, Sara D. Ladrón de Guevara, Veracruz, Universidad Veracruzana.

Universidad Veracruzana (2017b), Estatuto de personal académico, Xalapa, Graphos, UV.

Universidad Veracruzana (2017c), "Reglamento del Programa de Estímulos al Desempeño del Personal Académico", Veracruz, Universidad Veracruzana, <https://www.uv.mx/dgdaie/files/2017/02/ REGLAMENTO-PEDPA-15-Febrero.pdf > [Consulta: diciembre de 2017].

Universidad Veracruzana (2016), "III Informe de Actividades 2015-2016, Dra. Sara Ladrón De Guevara", en Documentos, Veracruz, Universidad Veracruzana, <https://www.uv.mx/universidad/ doctosofi/informe2015-2016/3-Informe-deActividades-2015-2016.pdf> [Consulta: enero de 2018]. 
Universidad Veracruzana (1994), Estatuto del Personal Académico, Xalapa, Graphos, UV.

Vargas, Luz (1994), "Sobre el concepto de percepción", Alteridades, vol. 4, núm. 8, pp. 47-53. <https://www. redalyc.org/pdf/747/74711353004.pdf> [Consulta: enero de 2019].
Vázquez, Lázaro (2012), "Los salarios de los trabajadores universitarios", Foro de Educación Superior, Revista Electrónica del Programa de Estudios Universitarios Comparados, núm. 22, <http://www.peu.buap.mx/web/ fes $/ 22 \% 20 \mathrm{FES} \% 20 \mathrm{Ano} \% 204 \% 20 \mathrm{No} \% 2022 / 03 \% 20$ El_salario_de_los_trabajadores_universitarios.pdf> [Consulta: diciembre de 2017].

\section{Cómo citar este artículo:}

Ocampo-Gómez, Elizabeth, Silvia Jiménez-García y Lilia Palacios-Ramírez (2020), "El investigador fragmentado: conflictos y tensiones derivados de la diversificación de su carga laboral”, Revista Iberoamericana de Educación Superior (RIES), vol. XI, núm. 27, pp. 41-56, https://doi.org/10.22201/iisue.20072872e.2020.30.587 [Consulta: fecha de última consulta]. 